\title{
PUBLICIDAD DE LAUDOS ARBITRALES EN EL ARBITRAJE COMERCIAL: ORDENANDO LA DISCUSIÓN
}

\section{PUBLICATION OF ARBITRAL AWARDS IN COMMERCIAL ARBITRATION: ORDERING THE DISCUSSION}

\author{
Rodrigo Zamora Etcharren \\ Galicia Abogados \\ Paola Aldrete Rivas* \\ Galicia Abogados \\ Santiago Oñate Yáñez ${ }^{* *}$ \\ Universidad Iberoamericana \\ Galicia Abogados \\ Adriana Lozano Cázares ${ }^{* * \star}$
}

Instituto Tecnológico y de Estudios Superiores de Monterrey

In recent years, the discussion regarding the publication of arbitral awards has been developing with several arguments being made for and against this type of measures. Multiple arbitration centers have adopted regulations about the subject in their latest rules. However, we still cannot determine which route commercial arbitration will follow when in regards to this matter.

In the following article, the authors evidence the multifaceted character of the discussion about the publication of arbitral awards in commercial arbitration. First, they present the regulations that the ten most relevant arbitration centers have adopted in regards to this subject. Then, they explain the characteristics of default rules and the consequences these can have depending on whether they are opt-in or opt-out. Finally, they explore the arguments in favor and in opposition to the publication of arbitral awards.

KEYWORDS: Advertising of arbitral awards; arbitral awards; default rules; double-hatting; commercial arbitration.
En los últimos años, la discusión sobre la publicidad de los laudos arbitrales se ha venido desarrollando y múltiples centros de arbitraje han adoptado regulaciones sobre dicho aspecto en sus reglamentos más recientes. Sin embargo, todavía no se ha determinado cuál es el rumbo que tomará el arbitraje comercial con relación a este tema.

En el presente artículo, los autores evidencian el carácter polifacético de esta discusión en el arbitraje comercial. Para ello, primero exponen las regulaciones que las diez instituciones arbitrales más importantes han adoptado con relación a la publicidad de laudos. De ahí, explican las características de las reglas default y las consecuencias que estas tienen dependiendo de si son opt-in u opt-out. Finalmente, abordan los argumentos a favor y en contra de volver públicos los laudos o ciertos elementos de los mismos.

Palabras Clave: Publicidad de los laudos arbitrales; laudos arbitrales; reglas default; double-hatting; arbitraje comercial.

* Abogado. Master of Laws (LL.M.) por la New York University. Ex Presidente del Comité de Arbitraje Comercial de la Barra Mexicana y segundo vicepresidente de la Junta de Gobierno de esta institución. Socio de Galicia Abogados (Ciudad de México, México). Contacto: rzamora@galicia.com.mx.

** Abogada. Master of Laws (LL.M.) por la Georgetown University. Asociada sénior de Galicia Abogados (Ciudad de México, México). Miembro fundador de Mexico City Very Young Arbitration Practitioners (MXVYAP) y de la Georgetown Law Association of Mexicans. Contacto: paldrete@galicia.com.mx.

*** Abogado. Master of Laws (LL.M.) por la Universidad de Yale. Profesor de la Universidad Iberoamericana. Asociado de Galicia Abogados (Ciudad de México, México). Contacto: sonate@galicia.com.mx.

${ }^{* * * *}$ Egresada de la Facultad de Derecho del Instituto Tecnológico y de Estudios Superiores de Monterrey. Asistente legal en Galicia Abogados (Ciudad de México, México). Contacto: alozano@galicia.com.mx

Nota del Editor: El presente artículo fue recibido por el Consejo Ejecutivo de THËMIS-Revista de Derecho el 13 de marzo de 2020, y aceptado el mismo el 2 de julio de 2020. 


\section{INTRODUCCIÓN}

La evolución del arbitraje comercial en los últimos años ha respondido a diversos cambios sociales, comerciales y legales, así como a distintas propuestas y facetas del gremio arbitral. Una propuesta de gran impacto, que sugiere no solo un replanteamiento de la práctica cotidiana del arbitraje sino un reentendimiento de sus alcances, es el de la publicación de los laudos arbitrales. Si bien no es una discusión estrictamente novedosa, consideramos que la misma amerita su compilación y orden.

Con este propósito, planteamos este tema de la siguiente manera. En primer lugar, se hace una exposición de las diez instituciones arbitrales más relevantes y la manera en que han decidido regular la publicidad de los laudos. En segundo lugar, para entender los efectos, naturaleza y alcance de dichas regulaciones, se presenta las características de las reglas default y las consecuencias de que la publicación de los laudos, en las distintas regulaciones, sea de carácter opt-in u opt-out. Subsecuentemente, se aborda diversos argumentos a favor y en contra de la publicidad de los laudos. En este sentido, nuestra propuesta y propósito es dual. Por un lado, atendemos el tema desde un punto de vista adjetivo y demostramos los alcances que tienen las reglas dispositivas (reglas default) como herramientas para moldear o sugerir las decisiones que tomarán las partes sobre la publicidad de los laudos. En segundo lugar, se resalta los aspectos sustantivos del tema al exponer los argumentos en contra y a favor de la publicación de los laudos arbitrales.

La intención del presente artículo es plantear que la adopción de políticas institucionales que abracen la publicidad de los laudos arbitrales o que las rechacen, no es un tema binario; sino multifacético. Con ello, se busca demostrar que una decisión al respecto primero debe contemplar el diseño de la regla default sobre la materia y los empujes o nudges que con la misma se quieran crear y, en segundo lugar, presentar los dos lados de cada postura para evidenciar la heterogénea composición del tema. En este sentido, no se defiende una postura en específico; sino que únicamente se presenta los elementos que consideramos deben tomarse en cuenta para la determinación del rumbo del arbitraje comercial, con relación a la publicidad de los laudos.

\section{LA REGULACIÓN DE LA PUBLICIDAD DE LAUDOS EN DISTINTAS INSTITUCIONES Y REGIONES}

La confidencialidad ha sido entendida como una de las principales ventajas para los usuarios del ar- bitraje. No obstante, dicha característica, en especial la confidencialidad de los laudos, ha sido objeto de críticas tanto dentro de la comunidad arbitral como fuera de la misma. Entre varias propuestas realizadas por diferentes abogados y académicos del medio, surge una que parece ser de particular interés: la publicación de laudos arbitrales comerciales. Mientras algunas instituciones arbitrales han incorporado en cierta medida las observaciones externadas en relación con este tema, otros reglamentos aún carecen de regulación particular al respecto. En este apartado se podrá encontrar de manera concisa la forma en que dicho tema se encuentra regulado en reglamentos de diversas instituciones arbitrales.

\section{A. Cámara de Comercio Internacional}

Si bien el reglamento vigente de la Cámara de Comercio Internacional (en adelante, $\mathrm{CCl})^{1}$, no contiene disposiciones en las que se regule la publicación de información o documentos relacionados con el procedimiento arbitral, la Nota a las Partes y al Tribunal Arbitral sobre la Conducción del Arbitraje de Conformidad con el Reglamento de Arbitraje de la $\mathrm{CCl}$ (en adelante, la Nota) emitida con fecha 1 de enero de 2019, sí lo hace. Es importante mencionar que desde la Nota del 30 de octubre de 2017 se preveía la publicación de información relativa a los tribunales arbitrales de los casos registrados después del 1 de enero de 2016. Sin embargo, no fue hasta la Nota del 2019 que se incluyeron las disposiciones relacionadas con la publicación de laudos.

Mediante dicha Nota, la $\mathrm{CCl}$ resalta que, para garantizar que el arbitraje siga siendo una herramienta de confianza para facilitar la actividad comercial, es fundamental aumentar la disponibilidad de información para las partes, la comunidad empresarial y las entidades académicas (2019, párr. 34). En este sentido, salvo acuerdo en contrario de las partes, la $\mathrm{CCl}$ publica en su sitio web, la siguiente información relativa a los arbitrajes registrados desde el 1 de enero de 2016: (i) los nombres de los árbitros; (ii) su nacionalidad; (iii) su posición dentro del tribunal; (iv) la forma de su designación; y, (v) si el arbitraje está pendiente de resolución o concluido. Lo anterior, sin publicar el número de referencia del arbitraje ni los nombres de las partes. Adicionalmente, dicha Nota prevé que respecto de los arbitrajes registrados desde el 1 de julio de 2019, la Corte también publicará (vi) el sector industrial relativo; y, (vii) los asesores jurídicos que representan a las partes (2019, párr. 36).

El cual entró en vigor el 1 de marzo de 2017. 
Por otro lado, la Sección III.D. de dicha Nota prevé que la Secretaría informará a las partes y árbitros, en el momento de notificación de cualquier laudo final dictado a partir del 1 de enero de 2019, que dicho laudo o cualquier otro laudo y opiniones divergentes o coincidentes emitidas dentro del procedimiento podrán publicarse de manera íntegra al transcurrir por lo menos dos años de la fecha de notificación. Este plazo de publicación puede modificarse por las partes. Asimismo, las partes pueden oponerse a la publicación o pedir que se anonimice el texto antes de su publicación.

\section{B. Comisión de las Naciones Unidas para el Derecho Mercantil Internacional}

El reglamento vigente de la Comisión de las Naciones Unidas para el Derecho Mercantil Internacional (CNUDMI) ${ }^{2}$ establece en su artículo 34 que un laudo podrá hacerse público solo bajo tres supuestos: (i) con el consentimiento de las partes; (ii) cuando una parte tenga la obligación jurídica de darlo a conocer; o, (iii) con motivo de un procedimiento legal ante un tribunal o autoridad competente.

Si bien esta disposición rige los arbitrajes comerciales que se lleven a cabo conforme a este reglamento, cabe recordar que mediante Resolución aprobada por la Asamblea General el 16 de diciembre de 2013, tomando en cuenta que dicho reglamento se usa ampliamente en la resolución de disputas inversionista-estado y reconociendo la necesidad de disposiciones sobre transparencia en la solución de tales controversias las cuales involucran el interés público, se aprobó la publicación de la Convención de las Naciones Unidas sobre la Transparencia en los Arbitrajes entre Inversionistas y Estados en el Marco de un Tratado.

Como su nombre lo indica, dicha convención solo es aplicable a arbitrajes entre inversionistas y Estados en el marco de un tratado de protección de inversiones ${ }^{3}$. Sin embargo, resulta interesante apuntar que éste prevé la publicación de información y documentos relacionados con el procedimiento tales como: nombre de las partes, notificación de arbitraje, respuesta a la misma, memorial de demanda y de contestación, órdenes procesales, decisiones y laudos del tribunal arbitral, entre otros.

\section{Centro Internacional para la Resolución de Disputas}

De conformidad con el artículo 30 inciso 3 del reglamento vigente del Centro Internacional de
Resolución de Disputas (por sus siglas en inglés, ICDR), un laudo de un procedimiento administrado bajo dichas reglas solamente podrá ser publicado con el consentimiento de todas las partes o si lo requiere la ley (2014). De acuerdo con esta misma disposición, como excepción, el administrador podrá publicar o hacer accesibles laudos, órdenes, decisiones y resoluciones que se hayan hecho públicos durante el procedimiento. Asimismo, salvo pacto en contrario de las partes, el administrador podrá publicar laudos, órdenes, decisiones y resoluciones seleccionadas que hayan sido editadas para ocultar los nombres y otros datos de las partes que permitan su identificación. Tanto el reglamento de 2005 como el de 2009 regulaban la posible publicación de laudos de la misma manera que el reglamento vigente.

\section{Centro Internacional de Arbitraje de Hong Kong}

De acuerdo con el artículo 45 del reglamento vigente del Centro Internacional de Arbitraje de Hong Kong (en adelante, por sus siglas en inglés, HKIAC), a menos que sea acordado por las partes, ninguna de ellas o sus representantes, así como el tribunal arbitral, cualquier árbitro de emergencia, perito, testigo o secretario de tribunal, podrá revelar o publicar cualquier tipo de información relacionada con el arbitraje, laudo o Decisión de Emergencia emitida en el arbitraje (2018). No obstante, en el mismo artículo se establece que el HKIAC podrá publicar cualquier laudo (completo, resumido o un extracto de) si se cumplen los siguientes aspectos: (i) se elimina toda referencia al nombre de las partes y cualquier información de identificación de las mismas; $y$, (ii) durante el plazo fijado por la HKIAC, ninguna de las partes objeta su publicación. En caso de existir objeción por alguna de las partes, el laudo no puede ser publicado. En particular, la página de preguntas frecuentes de la HKIAC agrega un requisito adicional: que una solicitud de publicación sea hecha a la HKIAC.

El reglamento del HKIAC de 2008 establecía los mismos requisitos para la publicación que el reglamento de 2018. No obstante, resulta importante recalcar que tanto en el reglamento del 2008 como el del 2013, se preveía como requisito adicional el que una solicitud de publicación fuera dirigida a la HKIAC. Este requisito fue eliminado en el reglamento de 2018. Sin embargo, como ya se comentó, la página de preguntas frecuentes de la HKIAC sigue mencionando dicho requisito.

Reglamento de Arbitraje de la Comisión de las Naciones Unidas para el Derecho Mercantil Internacional con el nuevo artículo 1, párrafo 4, aprobado en 2013.

3 Conforme a su artículo 1, dicho reglamento es aplicable a arbitrajes entablados en virtud de tratados de inversiones celebrados a partir del 1 de abril de 2014 . 


\section{E. Corte de Arbitraje Internacional de Londres}

El artículo 30.1 del reglamento vigente de la Corte de Arbitraje Internacional de Londres (en adelante, por sus siglas en inglés, $\mathrm{LCIA})^{4}$ establece como principio general que las partes se comprometen a mantener como confidenciales todos los laudos emitidos en el arbitraje; así como todos los demás materiales y documentos presentados o emitidos dentro del mismo. Adicionalmente, el artículo 30.3 recalca que la LCIA no publicará ningún laudo ni partes de un laudo sin el previo consentimiento por escrito de las partes y del tribunal arbitral.

Lo dispuesto en dichos artículos se encuentra confirmado en la sección 19 de la Nota para las Partes de la LCIA, la cual establece que la LCIA no publicará laudos o secciones de laudos, incluso si se suprime información de identificación de los mismos. Sin embargo, esta misma sección agrega que la LCIA podrá publicar extractos de decisiones tomadas por la Corte en relación con recusaciones de árbitros, así como información estadística sobre casos.

\section{F. Centro Internacional de Arbitraje de Singapur}

El reglamento vigente del Centro Internacional de Arbitraje de Singapur (en adelante, por sus siglas en inglés, SIAC) ${ }^{5}$ establece de manera expresa en su artículo 39 que, salvo pacto en contrario de las partes, cualquier parte, árbitro, árbitro de emergencia, persona designada por el tribunal arbitral, incluyendo secretarios administrativos y peritos, deberán tratar todos los aspectos relacionados con el procedimiento y el laudo como confidenciales. En este sentido, si bien se prevé este principio de confidencialidad, bajo las reglas del SIAC sí se pueden publicar los laudos, siempre y cuando exista consentimiento de las partes y el tribunal arbitral.

Ahora, aunque en los reglamentos de 2007 y 2010 no se reguló respecto a la publicidad de los laudos, en el reglamento de 2013 se estableció que el SIAC podía publicar cualquier laudo suprimiendo los nombres de las partes y otra información relevante. No obstante, en el reglamento de 2016 se precisó que solo se puede realizar la publicación de laudos si se cumple con este requisito y, adicionalmente, si cuenta con el consentimiento de las partes y el tribunal arbitral (SIAC, 2016, art. 32.12).

\section{G. Instituto de Arbitraje de las Cámaras de Suiza}

Como regla general, en procedimientos sustanciados bajo el Reglamento Suizo de Arbitraje Internacional (en adelante, por sus siglas en inglés, SRIA) ${ }^{6}$, las partes, los árbitros, los peritos nombrados por el tribunal, el secretario del tribunal, los miembros del Consejo Directivo de la Institución de Arbitraje de las Cámaras Suizas y los miembros de la Corte y la Secretaría, se comprometen a mantener confidenciales todos los laudos y las órdenes, así como todos los documentos presentados por las partes en el marco del procedimiento.

Sin embargo, el artículo 44 inciso 3 establece que un laudo o una orden podrán ser publicados íntegramente, por extractos o de forma resumida solamente si se cumplen los siguientes aspectos: (i) una solicitud de publicación es remitida a la Secretaría; (ii) todas las referencias a los nombres de las partes son suprimidas; $y$, (iii) ninguna de las partes se opone a esta publicación dentro del plazo fijado por la Secretaría.

\section{H. Cámara de Comercio de Estocolmo}

Por su lado, la Cámara de Comercio de Estocolmo (por sus siglas en inglés, SCC) establece expresamente en el artículo 3 de su Reglamento ${ }^{7}$ que, salvo acuerdo en contrario de las partes, la Cámara, el tribunal arbitral y cualquier secretario administrativo deberán mantener la confidencialidad del arbitraje y del laudo.

\section{Centro de Arbitraje de México y Cámara Na- cional de Comercio de la Ciudad de México}

Aunque los reglamentos de ambas instituciones arbitrales carecen de regulación específica sobre la publicidad o confidencialidad del laudo emitido en dichos procedimientos, se puede desprender que los laudos emitidos son confidenciales; puesto que el reglamento del Centro de Arbitraje de México (en adelante, CAM) establece que todos los procedimientos arbitrales administrados bajo dichas reglas cuentan con dicho carácter ${ }^{8}$.

En este mismo sentido, la regulación establecida por el reglamento de la Cámara Nacional de Comercio de la Ciudad de México (en adelante, CANACO) es similar. El reglamento de la CANACO

\footnotetext{
Vigente a partir del 1 de octubre de 2014.

Vigente a partir del 1 de agosto de 2016.

Vigente a partir del 1 junio de 2012.

Vigente a partir del 1 enero de 2017.

8 Definición de "confidencialidad" prevista en el Reglamento de Arbitraje del Centro de Arbitraje de México en vigor a partir del 1 de julio de 2009.
} 
prevé que, salvo acuerdo en contrario de las partes, las actuaciones arbitrales serán confidenciales (2012, art. 5). Sin embargo, no reglamenta de manera particular la publicidad de los laudos.

Es importante precisar que, para efectos de este artículo, consideramos que la diferencia entre las instituciones que sí regulan el tema de la publicidad de laudos arbitrales y aquellas que no, es que las primeras incorporan explícitamente reglas de opt-in u opt-out. Es decir, establecen una regla default específica sobre la publicación y las condiciones para acordar la no publicación. Es en ese sentido que, si una institución no prevé presupuestos específicos sobre la publicidad de los laudos, los autores consideramos que esto último no está regulado, sin que ello sea óbice para considerar que en la práctica esta ausencia de regulación implícitamente conlleva una determinación sobre la no publicidad de los laudos arbitrales.

\section{LAS REGLAS DISPOSITIVAS O DEFAULT Y SU NATURALEZA}

La regulación antes descrita demuestra mucho más que la previsión (o falta de esta) respecto de la publicidad de laudos, por parte de las instituciones arbitrales. Es muestra de una proposición sobre el carácter que se le quiere dar a los laudos arbitrales, en específico, y al arbitraje, en general. Para ello, y sin ánimos de entrar en discusiones teóricas que escapan la materia del presente artículo, es importante entender de forma general el alcance y naturaleza de las reglas default. Ello porque las reglas sobre publicidad de laudos arbitrales son en esencia reglas default y porque la tendencia hacia una política de transparencia o de confidencialidad estará en gran medida definida por el tipo de regla default que adopten las instituciones arbitrales.

Las reglas dispositivas o mejor conocidas como reglas default son inspiradas por la teoría del contrato completo (theory of complete contract), la cual bajo una aproximación económica propone que estas reglas deben ser espejos de las preferencias de las partes y con ello rellenar las lagunas inevitables en el derecho contractual o en cualquier otra transacción (Bender, 2020, p. 5).
En este sentido, se ha considerado que las reglas default deben cumplir con dos requisitos: (i) la posibilidad formal de una exclusión voluntaria (optout), la cual conlleva una posibilidad para que las partes puedan manifestar preferencias ${ }^{9}$; y, (ii) el análisis de quién es el evasor de menor costo. Este último concepto si bien ha sido desarrollado principalmente en la responsabilidad extracontractual en los Estados Unidos de América, tiene aplicabilidad a un sinnúmero de situaciones sociales y jurídicas. Esencialmente, implica que se debe adjudicar la responsabilidad a quien esté en mejor posición para minimizar los costos de la decisión. Este concepto puede ser analizado o entendido desde distintos ángulos y uno de ellos podría argumentarse que está relacionado con quién tiene las mejores capacidades para establecer pautas de conductas y acciones. En el caso que nos ocupa, si la institución arbitral es quien establece la regla default, se entiende que son éstas las que mejor posicionadas están para minimizar los costos de la decisión.

Asimismo, las reglas default además de fungir como representaciones estándar de preferencias, también sirven en su diseño como mecanismos de empuje (nudges). De tal manera que, con su inclusión, se impulsa a las partes hacia comportamientos específicos. Dicho aspecto lo podemos ver ejemplificado con el hecho de que al incrementar los costos de la exclusión voluntaria (opt-out) de una regla default, gradualmente se puede incrementar la apariencia de las reglas default como reglas obligatorias (Bender, 2020, p. 21; Sunstein, 2013 , p. 35). El incremento de los costos en la exclusión voluntaria se puede ver reflejado en las diversas reglas secundarias que acompañan la regla default (requisitos para publicar el laudo, formas de modificar la publicación, etc.). En este sentido, quien establece las reglas default también sienta una pauta de conducta ${ }^{10}$.

Otro aspecto que subyace en la creación de reglas default es la minimización de costos transaccionales (Ayres, 2012, p. 2054). Si bien el costo transaccional de la decisión sobre publicidad de laudos pudiera parecer mínimo o inexistente, éste sí representa un elemento de cierta importancia

$9 \quad$ Ello porque de no adecuarse las reglas default a las preferencias, las personas buscarían formas de acordarlas por fuera de sus parámetros contractuales, institucionales, reglamentarios, etc. Dicho aspecto incrementaría, de forma inevitable, los costos transaccionales.

10 Para poder definir una regla default, se deben contestar las siguientes preguntas: (i) ¿debería ser cierta regla obligatoria o contratable?; (ii) si es contratable, ¿cuál debería ser el default?; y, (iii) si es contratable, ¿cómo se puede contratar alrededor de la regla default? Lo que Ayres ha denominado como altering rules, ya que se alteran las consecuencias legales de la regla. El proceso de opt-out es a la vez un proceso de opt-in a consecuencia que no son de default. Las altering rules pueden ser creadas de distintas formas y para distintos propósitos, por un lado, pueden dificultar la opción de contratar o acordar fuera de la regla default o, por otro lado, pueden llegar al punto de nulificar la posibilidad. Ambas pueden ser estrategias para disminuir ciertos errores, homologar ciertas conductas o proponer una política específica (Ayres, 2012, p. 2034). 
al analizar los efectos de la regla. Ello se debe a que dicha regla le presenta a las partes una opción predeterminada, lo que disminuye los costos asociados con llegar a un acuerdo sobre una cuestión adicional al procedimiento arbitral.

Es en este contexto que tenemos que analizar las reglas de publicación expuestas en el apartado anterior. El entendimiento de la formulación de reglas de publicación debe atender a diversos elementos. En primer lugar, debemos advertir si se regula la publicidad de los laudos a través de la creación de reglas default o si, por el contrario, no se regula y las partes son libres de acordar lo conducente. En este último caso, no estaríamos ante el supuesto de las reglas default, ya que no existiría una regla preestablecida que las partes pudieran decidir acoger o rechazar, sino que ellas mismas tendrían que anticipar el tema y acordar sobre el mismo.

Ahora, en caso de ser las instituciones arbitrales quienes impongan una regla, estamos ante el fenómeno descrito de reglas default. Este escenario genera dos supuestos: (i) que la regla sea en el sentido de que los laudos serán publicados en tanto las partes no se manifiesten en contrario; o, (ii) que la regla sea en el sentido de que los laudos no serán publicados a menos que las partes soliciten su publicación. El primer escenario sería una regla default opt-out y el segundo, una regla default opt-in.

En el caso que nos ocupa y tomando en consideración que las reglas default evidencian ciertos valores que se intentan empujar, la regla default que establezca que los laudos serán públicos (opt-out) promovería valores de apertura y transparencia. En sentido contrario, una regla default que establezca que los laudos serán confidenciales a menos que las partes pacten lo contrario (opt-in) dotaría de mayor valor a principios como la voluntad de las partes y la confidencialidad en el arbitraje.

De tal manera, las condiciones o requisitos impuestos para el opt-out u opt-in, indicarán el grado de obligatoriedad aparente con la que se busca envestir a la regla y los costos de pactar fuera de ella. Así, las condiciones que acompañen esta decisión de las partes ${ }^{11}$ pueden ser creadas de distintas formas y para distintos propósitos. Por ejemplo, supongamos que una regla default en un reglamento de arbitraje prevé la publicidad de los laudos y las condiciones, reglas secundarias o requisitos, únicamente contemplan el alcance de dicha publici- dad (nombres de árbitros, nombres de las partes, montos, etc.). En este caso se revestiría a la regla default con una obligatoriedad más aparente, lo que posiblemente haría que las partes decidieran no eliminar por completo la publicidad; sino limitarse a modificar dicha regla con alguna de las opciones preestablecidas para su modulación. De tal forma, la flexibilidad de las reglas secundarias que engloben a la regla default también indican la firmeza del empuje o nudge que la institución quiere dar respecto a la publicidad.

En este sentido, el análisis y definición del tipo de regla default y los alcances de sus reglas secundarias nos dicen mucho sobre los efectos prácticos de su implementación.

\section{ARGUMENTOS A FAVOR DE LA PUBLICIDAD}

En primer término, analizaremos los diversos argumentos que podrían justificar una política de transparencia o publicidad respecto a los laudos arbitrales. Los autores hemos identificado ocho argumentos al respecto, estos son los siguientes: desarrollo del Derecho (costumbre y práctica); certidumbre y seguridad jurídica; consistencia; educación y aprendizaje (creación de doctrina); calidad de laudos; desmonopolización y legitimidad; equidad procesal entre jugadores primerizos y jugadores recurrentes; transparencia y anti-corrupción. Los argumentos identificados no pretenden ser una lista exhaustiva, pero sí consideramos que son representativos de los principales postulados a favor de la publicación de los laudos arbitrales.

\section{A. Desarrollo del Derecho (costumbre y práctica)}

A pesar de que la costumbre y usos comerciales influyen en gran medida en la aplicación del Derecho en los procesos arbitrales, la emisión de laudos arbitrales fundados funciona dentro del concepto más general sobre la aplicación sustantiva del Derecho. De tal manera que para que el arbitraje se desarrolle de forma creativa y propositiva, por un lado, se debe garantizar su esencia de resolución de controversias y, por el otro, se debe buscar que el mismo sirva como espacio fértil para la creación y elaboración de derecho sustantivo (Carbonneau, 1985, p. 586).

La noción de un derecho común arbitral ${ }^{12}$ se robustece con la idea de que las visiones Estado-

11 Este aspecto se ha definido como altering rules por Ayres (2012, p. 2034). Para mayor información, véase el art. 5 del Reglamento de CANACO.

12 La idea de Common Law del arbitraje no es exclusiva del common law, sino que puede ser vista como una consecuencia de la práctica arbitral internacional y la creación de cuerpos normativos de aplicación común. Al respecto, véase a Carbonneau (1985, p.586) 
céntricas sobre la creación de derecho comercial internacional se ven retadas con una realidad que pone de relieve la poca inclinación y, en algunos casos, la falta de competencia de los Estados para generar un Derecho que se adecúe a las exigencias y velocidad de las transacciones comerciales y sus usos (lex mercatoria) (Gopalan, 2004). En este sentido, es importante precisar que la armonización del derecho comercial no conlleva la implementación de un solo conjunto normativo sino que es una forma de acomodar una serie de conceptos legales en distintas jurisdicciones (Zamora, 1995; Gopalan, 2004).

Con ello se resalta la importancia del desarrollo del derecho arbitral. Si bien es cierto que los tribunales arbitrales aplican, en la mayoría de los casos, un derecho sustantivo de un país en específico, no menos cierto es que con independencia de ese derecho sustantivo, crean un corpus iuris propio sobre el procedimiento $y$, en algunos casos, sobre la interpretación de prácticas comerciales. El arbitraje internacional comercial carece de una teoría del precedente, sin embargo, se han identificado tendencias que apuntan a que los tribunales arbitrales hacen referencia en sus laudos a otras resoluciones (Kaufmann-Kohler, 2007). De tal manera, la publicación de laudos podría contribuir al desarrollo del derecho arbitral (Zlatanska, 2015, p. 28), así como de la lex mercatoria, promoviendo con ello la deslocalización del arbitraje ${ }^{13}$.

Por ende, la publicidad de los laudos ayudaría a generar una teoría del precedente sólida, que permitiera un desarrollo jurídico de la materia sobre la base de una creación constante por los tribunales arbitrales. Lo que también llevaría inevitablemente al siguiente punto, la certidumbre y seguridad jurídica.

\section{B. Certidumbre y seguridad jurídica}

Tomando en consideración la tendencia de los tribunales arbitrales descrita anteriormente por usar, citar y referirse a otros casos, la publicación de los laudos promete: primero, solidificar una teoría y práctica del precedente; y con ello, posteriormente, generar certidumbre jurídica a los usuarios del arbitraje. Esta certidumbre no debe entenderse en el sentido más tradicional del ámbito judicial, sino como una certidumbre dentro de las prácticas comerciales y su constante y evolutiva interpretación por tribunales arbitrales.
Esta certidumbre no se agota únicamente en las interpretaciones de prácticas comerciales, reglas procesales y jurisdiccionales, sino que también podría servir para generar certidumbre y seguridad jurídica respecto a las decisiones de árbitros en específico. Dicha cuestión será abordada con mayor detalle en la siguiente sección.

Ambas razones persiguen una finalidad económica; la previsibilidad de resultados. Esto es esencial en el comercio internacional, ya que de ello dependen un sinnúmero de decisiones, inclusive un análisis de costo/beneficio respecto a la iniciación de un procedimiento arbitral (Zlatanska, 2015, p. 28). De forma específica, las partes podrán identificar y valorar (con anterioridad al inicio del arbitraje) casos similares en los que se hayan analizado contratos, situaciones y pretensiones parecidas a las suyas y así determinar las probabilidades de éxito de sus reclamos (Raymond, 2005, p. 479).

Ante ello, la preparación para un potencial caso conllevaría necesariamente el análisis de casos análogos, emulando hasta cierto punto la práctica del estudio de precedentes del common law. Sería impensable que un abogado en los Estados Unidos de América, antes de iniciar un litigio, no tuviera conocimiento sobre todos o la mayoría de los casos similares al de su cliente. Esto no solo exige de los abogados una preparación distinta, sino que también implica que los análisis de éxito presentados a sus clientes tendrán mayor certeza, ya que serán elaborados sobre la base de casos concretos. En la actualidad, la falta de publicidad de laudos ocasiona que los abogados transfieran este tipo de información de "boca en boca", o, en algunos casos, que sean las instituciones quienes determinen de cuáles casos se publican extractos que consideren relevantes, como lo hace actualmente la $\mathrm{CCl}$.

\section{Consistencia}

La consistencia presupone la uniformidad de ciertos elementos. En el caso del arbitraje internacional la publicidad de laudos, así como la propagación de una cultura jurídica del precedente, llevarían a que las reglas se aplicaran de una manera más uniforme. Aquí cabe precisar que el derecho aplicable al fondo de cada caso no debería verse afectado por esto, ya que las reglas de aplicabilidad de los precedentes claramente funcionarían bajo una racionalidad de derecho aplicable, así como las reglas procesales de la institución arbitral de la cual se tratase. En este sentido, se ha considerado que la

13 También denominado arbitraje "a-nacional" o "transnacional" implica que el arbitraje debería ser regulado por normas transnacionales que no se vieran afectadas por normas aplicables a procedimientos locales. Para mayor información, véase a González de Cossío (2014, p. 159). 
publicación de laudos llevaría al desarrollo y consistencia del derecho arbitral, ya que a pesar de que estos son vinculantes, únicamente entre las partes litigantes y los laudos bien razonados podrían tener un valor persuasivo que fomente una "sabiduría arbitral común", la cual iría más allá de un círculo privilegiado de abogados y firmas (Buys, 2004, p. 136) [traducción libre].

Ahora bien, es importante precisar que ya desde hace tiempo se había identificado, por ejemplo, en los laudos de la $\mathrm{CCl}$, que los aspectos sustantivos de los mismos mostraban señales del inicio de la gestación de un conjunto de principios jurídicos. Estos principios se originaban de una pluralidad de fuentes y leyes comerciales locales; sin embargo, se advertía cierta consistencia o por lo menos, aspiración de consistencia (Ayres, 2012, p. 589). Esto nos indica que la consistencia es un valor que busca obtenerse mediante la adjudicación en el arbitraje internacional y que, a través de la publicación de laudos, su consecución sería no solo más palpable sino más sistemática.

\section{Educación y aprendizaje (creación de doc- trina)}

Las tradiciones jurídicas que basan su aprendizaje en la creación casuística del derecho, dependen inevitablemente de las resoluciones que emitan sus órganos jurisdiccionales. Ello es una consecuencia natural y necesaria de un sistema de precedentes, ya que los estudiantes de derecho, así como los abogados postulantes, aprenden y se actualizan a través del análisis de las resoluciones. El arbitraje internacional, hoy en día, se acerca y asemeja en ciertos puntos a los sistemas de precedentes. Con la publicación de laudos se lograría tener un repertorio mucho más amplio y nutrido de casos que permitan observar y aprender sobre la evolución interpretativa de este mecanismo de adjudicación.

Habrá quienes opondrán que el arbitraje se encuentra enfocado en la solución de conflictos comerciales muy específicos y, por lo tanto, que su valor no es educativo sino meramente de resolución de disputas concretas. Sin embargo, al ser un mecanismo de solución de controversias y, con ello, de creación de derecho, no deja de ser un "fenómeno social" que debe ser estudiado (Smit, 2000, pp. 567 y 580)

Asimismo, la publicación de los laudos servirá para consolidar no solo la enseñanza del arbitraje, sino también la inculcación de una aproximación de "teoría del caso" a los problemas, especialmente para alumnos de tradiciones civilistas. En este mismo tenor, la enseñanza a través de los laudos serviría no solo para exponer la creación y aplicación de ciertos principios; sino también para demostrar las características de ciertas tradiciones jurídicas y sus métodos de razonamiento jurídico. Todo ello no se extingue en una finalidad puramente académica o pedagógica, sino que tendría un efecto práctico que no debe soslayarse: los abogados postulantes contarían con herramientas adicionales para argumentar y presentar sus casos.

\section{E. Calidad de laudos}

La práctica actual del arbitraje internacional respecto a la publicación de laudos se ha desarrollado en el sentido de que los nombres de los árbitros no se den a conocer. Sin embargo, bajo una política más transparente de publicidad, la revelación de nombres de árbitros llevaría probablemente a un mayor cuidado en la redacción de los laudos. Ello porque el foro para quienes escribirían dejaría de ser únicamente las partes, sino sería la comunidad arbitral y jurídica. En este sentido se ha pronunciado Smit al considerar que la publicación de los nombres de los árbitros garantizaría no solo una mayor calidad de los laudos arbitrales, sino que también estimularía o incentivaría a los árbitros a ser más creativos y novedosos en sus resoluciones (1991).

La publicación de los laudos, junto con los nombres de los árbitros, fungiría como un incentivo externo para motivar a los árbitros a desarrollar sus laudos con vistas a un público mayor. En este mismo sentido, debe resaltarse que otra consecuencia de lo anteriormente descrito, sería una nueva o regenerada valoración de los votos disidentes, ya que estos servirían también para fijar opiniones, posturas e inclusive, para nutrir el debate de ideas en el foro arbitral.

\section{F. Desmonopolización y legitimidad}

La legitimidad de las instituciones impartidoras de justicia conlleva un elemento ineludible de percepciones y justicia procesal. Desde la psicología y el análisis psicológico del Derecho, ha sido ampliamente estudiado el efecto que tienen las percepciones creadas en la sociedad en la legitimidad de las instituciones ${ }^{14}$. Estas percepciones se construyen de distintas formas y por una variedad de factores, siendo uno de ellos el acceso o la transpa-

14 Existen diversos ejemplos de intentos por sistematizar la práctica arbitral. Uno de ellos siendo el de la lex petrolea, que desde finales de los 90 s se empezó a erigir como un conjunto de reglas que gobernaban las relaciones y transacciones petroleras. Si bien este tema ha sido criticado y aproximado con escepticismo por algunos, es importante traerlos a colación. Al respecto, para mayor información, véase a Daintith (2017) y Mersadi Tabari (2016). 
rencia de las actuaciones. En este sentido, la publicación de los laudos arbitrales crearía una imagen de apertura mediante la cual se daría a conocer el cómo se decide y cómo funcionan los procedimientos arbitrales.

Así, si bien muchos podrían argumentar que el arbitraje debe restringirse a la solución de controversias específicas, no menos cierto resulta que el arbitraje también es un fenómeno social y por ello, sus efectos van más allá de casos específicos (Zlatanska, 2015, p. 29; Smit, 2000, pp. 567 y 580). En este sentido, entre mayor visibilidad tenga la labor de los árbitros, más robustecida se verá la legitimidad del orden arbitral y sus procesos. Asimismo, muy probablemente una medida de este tipo tendría el efecto de ganar más adeptos para el arbitraje, ya que la visibilidad y transparencia permitiría que las personas lo entendieran y vieran como un mecanismo de solución de controversias accesible y viable, no como un sistema lejano, oscuro y confuso.

Esta transparencia también ayudaría a visibilizar ciertas prácticas que pudieran considerarse como monopólicas en el arbitraje. Contribuiría al entendimiento de cuáles personas o grupos de personas cuentan con poder e influencia dentro del sistema (power brokers). Un fenómeno que ha llevado a múltiples análisis sobre la configuración del arbitraje internacional, así como potenciales conflictos de interés ${ }^{15}$. Este es un tema sin duda controversial, pero la transparencia del arbitraje promovería, por lo menos, el análisis sobre este tipo de prácticas.

\section{G. Equidad procesal entre jugadores primeri- zos y jugadores recurrentes}

Se podría decir que una de las ventajas del arbitraje radica en la posibilidad que tienen las partes de seleccionar a sus árbitros. Ello figura no solo como una característica de adjudicación flexible sino también como emblema de la centralidad que tiene la autonomía de las partes, con la excepción de casos en donde la institución arbitral es la que elige a los árbitros. Sin embargo, ello tiende a favorecer a las partes (entiéndase también abogados de parte) que ya han tenido experiencia con el arbitraje. Ello es así porque la información sobre árbitros es relativamente escaza (Zlatanska, 2015, p. 31). Esto lleva a que la información sobre árbitros e instituciones se obtenga a través de la práctica e interacción dentro de este foro. De tal manera que los jugadores recurrentes (vis-à-vis) los jugadores primerizos, tengan mayor ventaja a la hora de elegir árbitros, instituciones o inclusive, abogados.

La publicación de laudos arbitrales ayudaría a nivelar el acceso al arbitraje para partes no experimentadas. Propiciaría que los árbitros creen reputaciones públicas que vayan más allá de lo conocido en el gremio y que permita a cualquier parte obtener más información sobre sus perfiles al momento de tomar una decisión sobre su elección. Asimismo, permitiría que árbitros más jóvenes, y sin tanta reputación gremial, logren hacerse de una imagen pública que les permita su inserción a mayores arbitrajes y así, se abonaría a una práctica más inclusiva y plural.

\section{H. Transparencia y anti-corrupción}

La transparencia en el arbitraje ha sido identificada bajo tres grandes rubros: (i) transparencia organizacional; (ii) transparencia legal; $y$, (iii) transparencia procedimental. La primera se refiere a la transparencia de las instituciones arbitrales sobre su manejo de casos y decisiones. La segunda atiene a la publicación de laudos arbitrales. La tercera versa sobre la publicidad de los procedimientos y audiencias arbitrales (Pernt, 2017). Mientras que la primera es una transparencia de aspecto más interno o que atiene más a las partes, las últimas dos son transparencias externas y que, por tanto, atienden a intereses que pudieran tener no solo las partes sino terceros.

En el ámbito de las políticas públicas y diseño institucional se ha considerado que la transparencia y los esfuerzos anticorrupción tienen una relación estrecha y, en algunos casos, incluso indisoluble. Los complementos naturales a cualquier medida punitiva en contra de la corrupción son la transparencia y la rendición de cuentas ${ }^{16}$. Estas conclusiones son perfectamente extraíbles del ámbito público y aplicables a escenarios como el del arbitraje internacional. El combate a la corrupción en el ámbito del arbitraje internacional tiene dos caras: el combate a la corrupción a través del arbitraje y el combate a la corrupción dentro del arbitraje. En cuanto al primero, ante la intensificación del combate a la corrupción a nivel mundial, el arbitraje ha tomado un papel importante en la adjudicación de casos de corrupción (Gaillard, 2019, p. 19). En estos casos, más allá de los efectos específicos de los laudos y sus consideraciones, la publicidad de los mismos serviría un papel social de divulgación de

15 Si bien este tema ha sido analizado con mayor profundidad en el arbitraje de inversión, muchos de los temas tratados son aplicables a la práctica comercial por igual. Para mayor información, véase a Langford et al. (2017).

16 Para mayor información, véase a Rose-Ackerman y Truex (2012). 
casos de corrupción así como sus consecuencias, y pondría de relieve la capacidad de los tribunales arbitrales para garantizar y promover los valores de la comunidad internacional en los esfuerzos en contra de la corrupción (Gaillard, 2019, p. 19).

En cuanto al segundo elemento, la publicación de laudos contribuiría a la disminución de los casos de corrupción en el arbitraje. Ello porque al ser públicas las decisiones de los árbitros, el escrutinio sobre sus decisiones e inclusive, sus móviles, podrían ser evidentes. De tal modo que en potenciales casos en los que se emitieran laudos sin fundamento o con señales de clara arbitrariedad, éstos enfrentarían consecuencias más allá de las legales involucradas en juicios de nulidad, sería su reputación la que estaría en juego.

\section{ARGUMENTOS EN CONTRA DE LA PUBLICI- DAD}

Existen distintos contrapesos frente a los beneficios que hemos analizado que podrían resultar de la publicidad de los laudos, entre los que encontramos: (i) la vulneración de los derechos de protección de datos personales o confidenciales; (ii) el encarecimiento del procedimiento arbitral; (iii) la imposibilidad de crear una verdadera doctrina arbitral comercial; el aumento de conflictos de interés y de recusaciones de árbitros; y, (iv) el double-hatting frente a la publicidad de los laudos.

\section{A. Vulneración de los derechos de protección de datos personales, confidencialidad y pri- vacidad}

Respecto de la publicidad de los laudos, como parte de las posibles afectaciones negativas, se ha identificado la vulneración de la confidencialidad, privacidad y datos personales. La confidencialidad (inherente al arbitraje) ha sido arduamente analizada en distintos foros (Berger, 1992) ${ }^{17}$. Tradicionalmente, el arbitraje comercial se ha catalogado como confidencial y privado, siendo dichas cualidades un atractivo para quienes desean mantener su reputación intacta e información de negocio resguardada (por ejemplo, secretos técnicos e industriales, situaciones financieras o estrategias de negocio) (Kaczmarczyk \& Lam, 2019). Por ello, las partes de un acuerdo arbitral presumen y pueden tener una expectativa de confidencialidad ${ }^{18}$ y privacidad $^{19}$ en el procedimiento.

En la última década, la protección de los datos personales ${ }^{20}$ ha adquirido mayor relevancia a nivel internacional. La inclusión de datos personales en los laudos de arbitrajes comerciales, a diferencia de los de inversión, es común. Es entonces posible identificar a las partes y árbitros de un procedimiento arbitral mediante la lectura del laudo. Además, algunos de los datos personales incluidos en los laudos califican como sensibles y su revelación puede incluso arriesgar la integridad de su titular. Finalmente, en algunos países, los datos sobre los costos del arbitraje y honorarios de los árbitros califican como sensibles, por lo que su resguardo y tratamiento debe de ser aún mayor respecto de los datos personales no sensibles.

Durante la sustanciación del arbitraje, la revelación de datos personales y de información de carácter confidencial es común. Dada la confidencialidad y privacidad del arbitraje, la revelación entre las partes (incluyendo árbitros) no conlleva mayor problema para los usuarios del arbitraje. Sin embargo, las recientes tendencias de transparencia y publicidad amenazan con vulnerar los derechos de los titulares de esa información. En ciertos países la publicidad de datos personales sensibles (e.g. costos del arbitraje o honorarios de los árbitros), puede incluso poner en riesgo la integridad física de las partes o de los árbitros ${ }^{21}$.

La disociación de la información respecto de los titulares disminuye las probabilidades de vulneración de los derechos de protección y de confidencialidad analizados. Es por ello que, como medida de mitigación, algunas las instituciones arbitrales han optado por publicar laudos en formatos de extracto, resumidos, revisados y/o anonimizados (Fernández-Rozas, 2009). Sin embargo, alcanzar el grado de desagregación suficiente para imposibilitar la identificación de las partes y controversia, resulta laborioso y costoso.

En consecuencia, la confidencialidad cede ante los recientes estándares y exigencias de transparencia y publicidad que pretenden ser aplicados en el ar-

17 Distintos análisis sobre el alcance, límites y excepciones (e.g. del interés público) de la confidencialidad se han realizado a la luz de la ley del lugar del arbitraje, del reglamento de la institución arbitral aplicable, el texto de la propia cláusula arbitral, y de los acuerdos sobre confidencialidad alcanzados por las partes.

18 No revelación de la información sobre lo que acontece en el arbitraje.

19 La privacidad en el arbitraje se refiere a la restricción de presencia y acceso a las audiencias arbitrales.

20 Los datos personales son aquellos que permiten la identificación de su titular (e.g. nombre, domicilio, origen étnico, religión, etc.).

21 En México, la información financiera de individuos cobra una relevancia mayor para ser protegida, dada la situación inseguridad del país. 
bitraje comercial. Ello ha generado la crítica principal sobre la publicidad de los laudos: que aún y cuando el arbitraje está sometido a una presunción de confidencialidad, ésta se ve superada, afectada e invalidada por la publicidad de los laudos.

\section{B. Encarecimiento y prolongación del procedi- miento arbitral}

En el ámbito comercial, a diferencia del ámbito de inversión, los árbitros se enfocan en resolver los casos tomando en cuenta las herramientas proveídas por las partes y sin necesidad de preocuparse por la creación de un posible precedente (Blackaby, 2006). Es decir, los árbitros resuelven la controversia de la manera más adecuada y ajustada al caso, incluyendo, en específico, sin considerar la posibilidad de que su resolución sea citada o utilizada por otros árbitros en casos similares (2006). Actualmente, los árbitros en arbitrajes comerciales no tienen la responsabilidad ni la obligación de crear un corpus iuris (2006), por lo que su tarea únicamente consiste en resolver el caso en específico.

Con la publicidad del laudo, también se corre el riesgo de que éste funcione como medio para que los árbitros mejoren su reputación al lucir sus habilidades legales, pudiendo distraerse de su tarea principal: resolver imparcialmente el caso con las herramientas proveídas por las partes y conforme al derecho (2006). Lo que pudiera incrementar los tiempos de su emisión.

La publicidad de los laudos en arbitrajes comerciales, traería consigo la necesidad de que los árbitros inviertan tiempo adicional en el análisis y razonamiento de los laudos dado que deberán delimitar claramente sus aristas para evitar que los mismos sean citados incorrectamente en otros arbitrajes. Ese tiempo adicional necesariamente ocasionaría que la emisión del laudo sea retrasada, aumentando así los honorarios de los árbitros.

Adicionalmente, la tarea de disociación, resumen o extracción de información de los laudos generaría costos adicionales a las instituciones arbitrales, costos que serían trasladados a los usuarios del procedimiento. Es decir, la publicidad de los laudos causaría el aumento de los costos en el arbitraje comercial.

En conclusión, una de las razones por las cuales la publicidad de los laudos se ha visto criticada yace en el posible encarecimiento y prolongación del procedimiento arbitral como consecuencia de la necesaria inversión adicional de tiempo de los árbitros en el dictado de sus laudos y de la institución arbitral en la desagregación de información confidencial de los laudos.

\section{La imposibilidad de crear una verdadera doctrina arbitral}

Como crítica a los supuestos beneficios que resultarían de la publicidad de los laudos, relativos al desarrollo del Derecho, certidumbre y seguridad jurídica, y consistencia, hay quienes han señalado que dichos beneficios no tendrían el resultado alcanzado en los arbitrajes de inversión y que se pretenden alcanzar en los arbitrajes comerciales. Las razones de la postura en contra se analizan a continuación.

A diferencia del arbitraje de inversión, en el arbitraje comercial las decisiones sobre el fondo de la disputa no prometen causar un impacto importante en la comunidad arbitral, pues los árbitros (no necesariamente expertos en la ley aplicable) son quienes interpretan las circunstancias del caso considerando la ley aplicable, así como la información proporcionada por las partes y expertos. A manera de ejemplo, en el análisis y razonamiento del fondo de un caso, la interpretación de la legislación mexicana realizada por un árbitro de nacionalidad distinta no forzosamente sería seguida por un árbitro mexicano.

La crítica anterior no es aplicable respecto de cuestiones procesales (i.e. bifurcación del procedimiento), competencia del tribunal arbitral, lex mercatoria, interpretación de cláusulas patológicas, aplicación de principios generales del derecho y de derecho público (Bentolila, 2017). La interpretación de dichas cuestiones resulta más interesante $y$, por lo tanto, más útil, como precedente en arbitrajes comerciales (Blackaby, 2006). Algunas instituciones arbitrales (como la $\mathrm{CCl}$ ) ya publican extractos de órdenes y resoluciones (incluyendo laudos) respecto de las cuestiones anteriores.

\section{Aumento de conflictos de interés y de recu- saciones de árbitros.}

Incluso cuando la publicidad de los laudos promete una equidad procesal entre jugadores primerizos y jugadores repetidos, por las razones que a continuación se detallan, pudiera entenderse que la balanza se tildaría hacia los jugadores primerizos quienes tendrían menos riesgos de que la publicidad de los laudos aumente las causas para su recusación.

Actualmente, las partes se valen de la información proporcionada voluntariamente por los árbitros (después de realizar el análisis respectivo) y de la información que pueden obtener de "boca en boca" de otros miembros de la comunidad arbitral. Mediante la publicación de laudos arbitrales, las partes tendrían una fuente adicional de 
información para cuestionar la imparcialidad de los árbitros. En principio, proveer a las partes de mayor información no aparenta un problema para el gremio arbitral. Sin embargo, una de las posibles causales para recusación es que el árbitro haya tomado una postura respecto de algún aspecto del arbitraje. Al día de hoy, la mayoría de los árbitros han sido cuidadosos en sus publicaciones. De ser publicados los laudos, existirían más herramientas para que las partes promuevan recusación.

Conforme a las Directrices del International Bar Association (por sus siglas en inglés, IBA) sobre Conflictos de Intereses en Arbitraje Internacional, el árbitro (dependiendo de las circunstancias del caso) deberá revelar si "ha manifestado públicamente una posición específica respecto de algún aspecto del arbitraje mediante una publicación, un discurso o de cualquier otra forma" (2014, sección 3.5.2).

Si fuera implementada la publicidad de los laudos en los arbitrajes comerciales, las posiciones de los árbitros sobre ciertos aspectos serían públicas. En ese sentido, habrá quien argumente que aumentaría el número de revelaciones que deberán realizar los árbitros a este respecto y, de suceder ello, naturalmente incrementaría el número de recusaciones de árbitros.

\section{E. Inhibición del double-hatting frente a la pu- blicidad de los laudos}

En el arbitraje comercial el double-hatting ${ }^{22}$ no es visto necesariamente como algo negativo. Sin embargo, si la publicación de laudos es implementada en el ámbito comercial, el double-hatting podría generar por lo menos dos tipos de conflictos de interés: (i) conflictos sobre aspectos/cuestiones; $y$, (ii) conflictos por confusión de roles ${ }^{23}$.

Por un lado, el conflicto de aspectos ha sido definido como un sesgo respecto de la postura del árbitro sobre un tema sustantivo que le impide abordarlo o analizarlo con mente abierta e imparcial (Blackaby, 2006). Cuando el árbitro debe analizar el mismo aspecto/cuestión que, como abogado de parte ha analizado en otro caso, corre el riesgo de que resuelva de conformidad con el análisis alcanzado por éste cuando actuó como representante de parte.

Por otro lado, la confusión de roles puede incluir la tentación de emitir laudos que puedan ser citados por el propio árbitro en otro caso en el que actúe como representante de parte (Blackaby, 2006). Al margen de lo anterior, es posible que los árbitros se vean influenciados en sus decisiones de votos particulares por el hecho de que el laudo sea público; es decir, su voto se podría ver afectado por el propósito de mantener cierta imagen o postura. En ese sentido, aumentaría también el número de votos particulares y disidentes, práctica que hasta el día de hoy ha sido cuidadosamente manejada por los árbitros para fortalecer la ejecutabilidad de los laudos.

Conforme a lo anterior, la publicidad de los laudos puede conllevar el aumento de conflictos de interés sobre aspectos de fondo y por confusión de roles.

\section{CONCLUSIÓN}

Este artículo pone de relieve lo multifacética que es la discusión sobre la publicidad de los laudos arbitrales. En un primer lugar, el análisis de las reglas default institucionales al respecto demuestra no solo lo que representan; sino lo que implican las posturas tomadas por las instituciones arbitrales, así como los valores o políticas que se intentan propulsar y, con ello, las condiciones con las que se acompañan dichas reglas. Tomando en consideración la naturaleza de las reglas default, así como las consecuencias de que se prevean mecanismos de opt-in u opt-out, tenemos que el gremio e instituciones arbitrales siguen siendo un cuanto reticentes a la incorporación de la publicidad de los laudos como la regla default.

Ello es así porque de las instituciones arbitrales mencionadas en la primera sección de este artículo, se advierte que el $70 \%$ prevé que la regla de default sea la no publicación y que la posibilidad de publicar laudos se atenga mediante la figura de opt-in, con distintas variaciones y condiciones. Por otro lado, solamente el $10 \%$ (la $\mathrm{CCl}$ ) prevé una regla de opt-out, mientras que el otro $20 \%$ no regula estos aspectos. Asimismo, y sin entrar a los detalles de cada regulación en específico, en algunos casos como el del SRIA, diversas condiciones que acompañan a la regla default, dificultan la opción de contratar o acordar fuera de la regla default y se aumentan los costos transaccionales de hacerlo.

Ante ello, si del análisis de los beneficios contra los perjuicios sobre la publicidad de los laudos resultare que las ventajas son mayores y que, por lo tanto, se quisiera impulsar una política de publicación

22 Para los abogados de arbitrajes comerciales es posible actuar como árbitro en un caso y como representante de parte en otro. Las cuestiones de hecho y derecho tienden a ser sustancialmente distintas de un caso a otro y la decisión del árbitro es usualmente privada.

23 Dicho conflicto es conocido como issue conflict. 
de laudos arbitrales, se tendría que comenzar por cambiar la regla default a una de opt-out, de tal manera que se disminuyan los costos transaccionales de contratar por fuera de la regla, se aumente el efecto de empuje hacia dicha conducta, y se normalice o estandarice dicha práctica. Lo cierto es que mientras las instituciones no sean quienes cambien dicha regla, será más difícil concebir o, por lo menos, más tardado lograr que las partes litigantes fueran quienes (a través de la elección de la publicación) lograran cambiar la práctica común. En este sentido, la tendencia hacia una política de transparencia o de confidencialidad, estará en gran medida definidas por el tipo de regla default que adopten las instituciones arbitrales.

Por otro lado, consideramos que existen diversos argumentos que justifican la publicación de los laudos arbitrales, como lo son: el desarrollo del Derecho; la certidumbre y seguridad jurídica; consistencia; educación y aprendizaje; calidad de los laudos; desmonopolización y legitimidad; equidad procesal entre jugadores primerizos y jugadores repetidos; y transparencia y anti corrupción.

Estos aspectos positivos encuentran igualmente convincentes argumentos en contra, como los siguientes: vulneración de los derechos de protección de datos personales, confidencialidad y privacidad; encarecimiento y prolongación del procedimiento arbitral; imposibilidad de crear una verdadera doctrina arbitral; aumento de conflictos de interés y recusaciones de árbitros; e inhibición del double-hatting frente a la publicidad de los laudos.

La presentación de ambas posturas evidencia lo polifacético que es el tema y la multiplicidad de elementos y consecuencias aparejadas con cada postura. Por ello, consideramos que debemos salir de discusiones maniqueas en las que se abraza por completo y sin reservas una política de publicidad en el arbitraje y por el otro, en las que se rechaza a ultranza cualquier propuesta en favor de la transparencia. Entre más entendamos los aspectos positivos y negativos que conlleva la publicación de laudos, así como de los elementos que configuran su diseño institucional a través de las reglas default, más nos acercaremos al justo medio sobre la dirección y rumbo que deberá tomar el arbitraje comercial respecto a la publicidad de los laudos.

Asimismo, advertimos y señalamos que ambas posturas están fuertemente arraigadas en un entendimiento particular de la naturaleza, ventajas y propósitos del arbitraje comercial. De tal manera, al iniciar cualquier discusión sobre el particular, recomendamos se cuestione si la postura asumida recae en un mantenimiento del status quo, radica en la defensa de elementos que se consideran definitorios de la institución arbitral o si bien, se plantea de forma propositiva para el mejoramiento de la misma. ${ }^{\circ}$

\section{REFERENCIAS}

Ayres, I. (2012). Regulating Opt-Out. The Yale Law Journal, 121(8), 2032-2116. https://digitalcommons.law.yale.edu/ylj/vol121/iss8/1

Bender, P.M. (febrero de 2020). Limits of Personalization of Default Rules. Towards a Normative Theory (working paper del Max Planck Institute for Tax Law and Public Finance número 2020-02).

Bentolila, D. (2017). Arbitrators' Constraints in Arbitral Decision-Making. En Bentolila, D. (Ed.), Arbitrators as Lawmakers (Vol. 43, pp. 145194). Kluwer Law International.

Blackaby, N. (2006). Investment Arbitration and Commercial Arbitration (or the Tale of the Dolphin and the Shark). En Mistelis, L.A. \& Mathew Lew, J.D. (eds). Pervasive Problems in International Arbitration (Vol. 15, pp. 217233). Kluwer Law International.

Bullard González, A., \& de la Jara, J. M. (2018). Análisis psicológico del Derecho (2da ed.). Editorial Jurídica THĒMIS.

Buys, C. (2004). The Tensions between Confidentiality and Transparency in International Arbitration. American Review of International Arbitration, 14(121).

Carbonneau, T. E. (1985). Rendering Awards with Reasons: The Elaboration of Common Law of International Transactions. Columbia Journal of Transnational Law, (23), 579-614. https://elibrary.law.psu.edu/cgi/viewcontent. cgi?article=1297\&context=fac_works

Centro Internacional de Arbitraje de Hong Kong (2020). HKIAC Administered Arbitration 50 Questions \& Answers. https://www.hkiac.org/ arbitration/why-choose-hkiac/hkiac-administered-arbitration-faqs\#050

Daintith, T. (2017). Against 'lex petrolea'. The Journal of World Energy Law \& Business, 10(1),113. https://doi.org/10.1093/jwelb/jww036

Fernández-Rozas, J. C. (2009). Trayectoria y contornos del mito de la confidencialidad en el arbitraje comercial. Arbitraje: Revista de Arbitraje Comercial y de Inversiones, Centro Internacional de Arbitraje, Mediación y Negociación 
(CIAMEN), (2), 335-378. https://eprints.ucm. es/9258/1/TRAYECTORIA_Y_CONTORNOS_ DEL_MITO_DE_LA_CONFIDENCIALIDAD.pdf

Gaillard, E. (2019). The emergence of transnational responses to corruption in international arbitration. Arbitration International, (35), 1-19. https://doi.org/10.1093/arbint/aiz004

González de Cossío, F. (2014). Arbitraje. Editorial Porrúa.

Gopalan, S. (2004). New Trends in the Making of International Commercial Law. Journal of Law and Commerce, (23), 117-142. https://www. cisg.law.pace.edu/cisg/biblio/gopalan.html

Kaczmarczyk, M. \& Lam, J. (2019). Sociology of Commercial Arbitration: Tools for the New Times. Journal of International Arbitration, 36(6), 693-726.

Kaufmann-Kohler, G. (2007). Arbitral Precedent: Dream, Necessity or Excuse? The 2006 Freshfields Lecture. Arbitration International, 23(3), 357-378. https://www.arbitrationicca.org/media/4/77507134886347/media01231914308713000950001.pdf

Langford, M., Behn, D., \& Lie, R.H. (2017). The Revolving Door in International Investment Arbitration. Journal of International Economic Law, 20(2), 301-332. https://doi.org/10.1093/jiel/ jgx018

Mersadi Tabari, N. (2016). Lex Petrolea and International Investment Law. Routledge.

Pernt, V. (2017, 4 de marzo). How Much (More) Transparency Does Commercial Arbitration Really Need? Kluwer Arbitration Blog. http:// arbitrationblog.kluwerarbitration.com/ 2017/03/04/how-much-more-transparencydoes-commercial-arbitration-really-need/

Peter Berger, K. (1992). The International Arbitrators' Application of Precedents. Journal of International Arbitration, (9), 5-22.

Raymond, A. (2005). Confidentiality, in a Forum of Last Resort? Is the Use of Confidential Arbitration a Good Idea for Business and Society? American Review of International Arbitration, (16), 479.

Rose-Ackerman, S., \& Truex, R. (2012). Corruption and Policy Reform (Yale Law \& Economics Research Paper No. 444). https://papers.ssrn. com/sol3/papers.cfm?abstract_id $=2007152$
Smit, H. (1991). Substance and Procedure in International Arbitration: The Development of a New Legal Order. Tulane Law Review, (65), 1309-1322. https://www.trans-lex. org/128900/_/smit-hans-substance-andprocedure-in-international-arbitration:-thedevelopment-of-a-new-legal-order-65-tullrev1991-at-1309-et-seq/

(2000). Breach of Confidentiality as a Ground for Avoidance of the International Agreement. The American Review of International Arbitration, 11(4).

Sunstein, C. R. (2013). Deciding by Default. University of Pennsylvania Law Review, 162(1), 1-57. https://scholarship.law.upenn.edu/penn_law_ review/vol162/iss1/1

Tyler, T. (2006). Why people obey the law. Princeton University Press.

Zamora, S. (1995). NAFTA and the Harmonization of Domestic Legal Systems: The Side Effects of Free Trade. Arizona Journal of International and Comparative Law, (12), 401-428.

Zlatanska, E. (2015). To Publish, or Not To Publish Arbitral Awards: That is the Question... The International Journal of Arbitration, Mediation and Dispute Management, 81(1), 25-37.

\section{LEGISLACIÓN, JURISPRUDENCIA Y OTROS DOCU- MENTOS NORMATIVOS}

Cámara de Comercio Internacional [CCI], Nota a las Partes y al Tribunal Arbitral sobre la Conducción del Arbitraje de Conformidad con el Reglamento de Arbitraje de la $\mathrm{CCl}, 1$ de enero de 2019. https://cms.iccwbo.org/content/ uploads/sites/3/2017/03/ICC-Note-to-Partiesand-Arbitral-Tribunals-on-the-Conduct-of-Arbitration_spanish.pdf

Cámara de Comercio Internacional [CCl], Reglamento de Arbitraje, 1 de marzo de 2017. https://iccwbo.org/content/uploads/sites/3/2017/03/ICC-2017-Arbitration-and2014-Mediation-Rules-spanish-version.pdf

Cámara Nacional de Comercio de la Ciudad de México [CANACO], Reglamento de Arbitraje, 23 de agosto de 2012 https://www.ccmexico.com. $\mathrm{mx} /$ documentos/legislacion/arbitraje_comercial/reglamento_arbitraje_canaco.pdf

Centro de Arbitraje de México, Reglamento de Arbitraje, 1 de julio de 2009. https://camex.com. $\mathrm{mx} / \mathrm{wp} / \mathrm{wp}$-content/uploads/2017/11/reglasvigentes-espanol.pdf 
Centro Internacional de Arbitraje de Hong Kong [HKIAC], Reglamento de Arbitraje Administrado, 1 de noviembre de 2008. https://www. hkiac.org/sites/default/files/ck_filebrowser/ PDF/arbitration/HKIAC_AAR_EC.pdf

Reglamento de Arbitraje Administrado, 1 de noviembre de 2013. https://www.hkiac.org/ sites/default/files/ck_filebrowser/PDF/arbitration/2013_hkiac_rules.pdf

Reglamento de Arbitraje Administrado, 1 de noviembre de 2018. https://www.hkiac.org/ sites/default/files/ck_filebrowser/PDF/arbitration/2018_hkiac_rules.pdf

Centro Internacional de Arbitraje de Singapur [SIAC], Reglas SIAC, 1 de julio de 2007. https:// www.siac.org.sg/our-rules/rules/siac-rules-2007

Reglas SIAC, 1 de julio de 2010. https://www.siac. org.sg/our-rules/rules/siac-rules-2010

Reglas SIAC, 1 de abril de 2013. https://www.siac. org.sg/our-rules/rules/siac-rules-2013

Reglas SIAC, 1 de agosto de 2016. https://www. siac.org.sg/our-rules/rules/siac-rules-2016

Centro Internacional para la Resolución de Disputas [ICDR], Reglamento de Arbitraje Internacional, 1 de junio de 2014. https://www.icdr. org/sites/default/files/document_repository/ International_Dispute_Resolution_Procedures_Spanish_0.pdf
Comisión de las Naciones Unidas para el Derecho Mercantil Internacional [CNUDMI], Reglamento de Arbitraje de la CNUDMI (con el nuevo artículo 1, párrafo 4, aprobado en 2013), U.N. Doc. A/RES/68/109 (2014).

Convención sobre la Transparencia en los Arbitrajes entre Inversionistas y Estados en el Marco de un Tratado, U.N. Doc. A/Res/68/109 (1 de abril de 2014), https://uncitral.un.org/sites/ uncitral.un.org/files/media-documents/uncitral/es/transparency-convention-s.pdf.

Corte de Arbitraje Internacional de Londres [LCIA], Reglamento de Arbitraje, 1 de octubre de 2014. https://www.lcia.org//Dispute_Resolution_Services/lcia-arbitration-rules-2014. aspx\#Translations

Nota para las Partes, 18 de agosto de 2017 https://www.lcia.org/adr-services/lcia-notesfor-parties.aspx

Instituto de Arbitraje de la Cámara de Comercio de Estocolmo [SCC], Reglamento de Arbitraje, 1 de enero de 2017. https://sccinstitute.com/ media/220137/arbitration_rules_spanish_17_ web.pdf

International Bar Association [IBA], Directrices IBA sobre Conflictos de Intereses en Arbitraje Internacional, 23 de octubre de 2014.

Swiss Chambers Arbitration Institute [SCAI], Reglamento Suizo de Arbitraje Internacional, 1 junio de 2012. https://www.swissarbitration.org/ files/33/Swiss-Rules/SRIA_spanish_2012.pdf 